\title{
Rate Monotonic Scheduling Analysis for Stability Constraints in Network Control System
}

\author{
Dr. N. Kalaiarasi ${ }^{1}$, Kalaivani C T ${ }^{2}$ \\ I'Professor, RMK College of engineering and technology, Chennai, INDIA) \\ ${ }_{2}^{2}$ (Research scholar, Saveetha Engineering College, Anna University, Chennai, INDIA,
}

\begin{abstract}
The Feedback control system connected to a real time network through which the control process takes place are called network control system(NCSs).Features of NCS is the information like reference input, plant output, control input is exchanged between sensors, controllers, actuator using a network like CAN bus. The system wiring is reduced, easy to maintain and increases the system reliability. In the conventional control theories uses some ideal assumptions like equal distance sensing, synchronized control and non-delayed sensing and actuation. But in time varying systems this assumption fails due to transmission delay or packet dropout. In this paper Stability of NCS is analysed by considering transmission delay for a computer controlled system using scheduling algorithm
\end{abstract}

Keywords : Network Control System, Real Time System, Stability

\section{Introduction}

The In direct digital control systems, computerized control replaced all analog instrumentation for the process control. Example system is shown in the figure 1 with a summary of several modular manufacturing system integrated control networks like FireWire, DeviceNet and Ethernet. In the example system, FireWire are used for the job requires high data throughput and high bandwidth. DeviceNet are used for the jobs which require low bandwidth like short, periodic messages signals transmission. Ethernet is preferred for the job like information exchange between the upper supervisory workstation and the controller is high volume but not time critical.

Sensors with analog outputs and actuators with analog inputs are point to point connection with the digital computers. Sensing, actuating and control signal are deadline based since speed of all digital control $\mathrm{CNC}$ machines are high due to accuracy of the product manufacture in the system. Some of the constraints in the design of the system which affects the performance is choosing good scheduling algorithm, either constant or time varying network induced delay between the sensor to controller and controller to actuator in the shared medium, unreliable transmission path, packet dropouts due to packet size, bandwidth and busy path. Depending on the systems environmental condition while analysing some parameter are kept constant. Fundamental issues like time varying transmission period, network scheduling, network induced delay, single packet or multiple packet transmission of plant inputs and output and dropping of the network packets are basic problems in NCSs. In this paper we have considered time delay factor as variable since message transmitted between devices are either control data, which is time critical or information data which only needs guaranteed delivery.

Figure 1: Real world NCS implementation

\section{Network Induced Time Delay}

The network induced delay in NCS occurs when sensors, actuators and controllers exchange data across the network. Destabilization and performance degradation even occurs due to network induced time delay. To avoid it most of the cases network messages are prioritized, higher priority messages are transmitted using CAN or DeviceNet by scheduling techniques. The Figure 2 illustrates how packets on random access networks are affected by random delays and the worst case transmission time of the packet is unbounded. The figure 3 shows how packet transmission delays occurs while waiting for the token or time slot on scheduling network. 


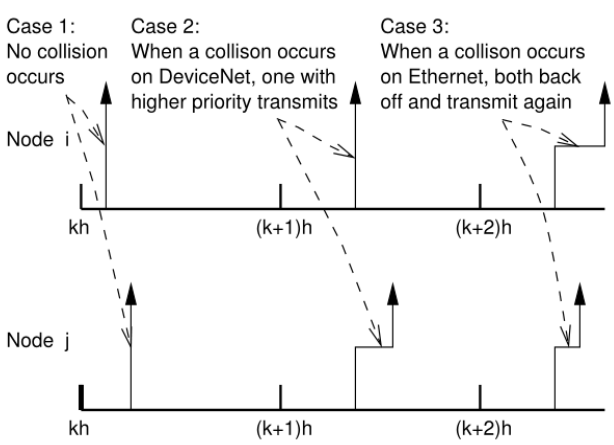

Figure 2: Time delay access between two nodes

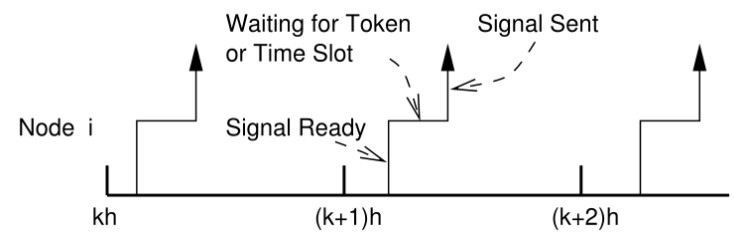

Figure 3: Time delay on scheduling network

\section{Analysics}

The system under study is shown in the figure 4. The NCS transmission period is denoted by $h(t)$

is defined as the time interval between two consecutive transmissions, measured between the instants when the transmission arrive i.e., arrival time. Ignoring the data processing overhead including building and queuing the data packet. Equal distance sampling cannot be guaranteed on NCS hence it is important to analyse the stability of NCSs when the sampling period is varying.

Case sensor to controller path is only considered with the assumption that transmission process is ideal i.e. no data processing time, scheduling time or transmission time. Hence, the sensors data is delivered instantaneously without delay. Further assumption is no packet loss during transmission.

\section{Network Control System Model}

In this paper we consider clock driven sensors that sample the plant outputs periodically or a periodically at sampling instant. Our goal in this paper is tofind the bound on the time varying transmission period when the feedback path is networked such that the NCS is still stable. We derive theorems for bounding the transmission period based on a variety of different methods.

Modelling NCS with time varying transmission period

The system equation of the network control system is

$\dot{x}(t)=A x(t)-B K \hat{x}(t), \quad t \in\left[t_{k}, t_{k+1}\right]$,

In terms of time delay

$$
\dot{x}(t)=\bar{A} x(t)-B K e(t)
$$

$\hat{x}\left(t_{k}{ }^{+}\right)=x\left(t_{k}\right), \quad k=0,1, \ldots \ldots$

Where $x(t) \in R^{n}$ is the state vector of the networked system, ${ }^{\hat{x}(t)}$ is piecewise continuous and only changes value at $t_{k_{z}}$ and $t_{k_{z}}, k=0,1, \ldots \ldots$ is the sampling instant or transmission instant. The figure 4 illustrates the trajectories of a state $^{\hat{x}(t)}$ and ${ }^{x(t)}$.e(t) be the transmission error or time delay between the states.

The equation is updated at the instant ${ }^{t_{k_{3}}}$ and kept constant until the next control update is received at time $t_{k+1}$ let $\mathrm{h}$ be the transmission period between successive transmissions.

$h_{k} \triangleq t_{k+1}-t_{k}$ 


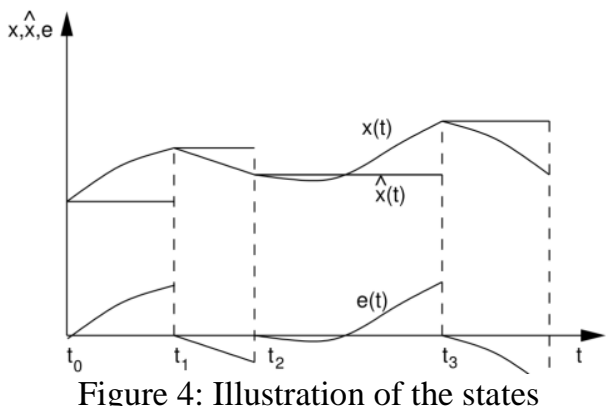

Let htrue denote the true bound on hk, and hence be a necessary condition. Hschur is the bound when constant transmission period is employed. To find, hsuff, is our goal still the system is exponentially stable.

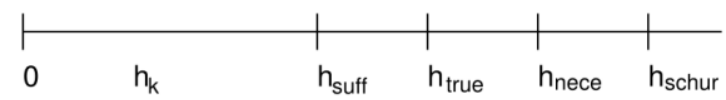

Figure 5: Bound of the transmission period

\section{Bellman-Gromwell Lemma}

To prove our theorems, we use Bellman -Gronwall lemma.

Lemma 1: let $\lambda(t)$ and $k(t)$ be nonnegative piecewise continuous functions of time and let $\lambda(t)$ be differentiable. If the function $\mathrm{y}(\mathrm{t})$ satisfies

$y(t) \leq \lambda(\mathrm{t})+\int_{t 0}^{t} k(s) y(s) d s \quad \forall t \geq t 0 \geq 0$,

Then

$y(t) \leq \lambda(\mathrm{t} 0) \exp \left(\int_{t 0}^{t} k(s) d s\right)+\int_{t 0}^{t} \lambda(\mathrm{s}) \exp \left(\int_{t 0}^{t} k(w) d w\right) d s, \quad \forall t \geq t 0 \geq 0$

Scheduling analysis

Each plant has a required transmission rate of their sensors data in order for it to be stable. when the transmission path is shared with the other NCSs transmission scheduling among the plants has to be considered. Formally, a schedule can be defined as a piecewise right continuous function $\sigma: R^{+} \rightarrow N$. An algorithm to find such a schedule $\sigma$ is called a scheduling algorithm.

Two problem are considered, schedulability analysis and optimal scheduling. network transmission are normally non pre-emptive. An on-going packet transmission cannot be interrupted due to a newly arrived transmission request. The figure 5 show the characteristics parameter of NCS data transmission.

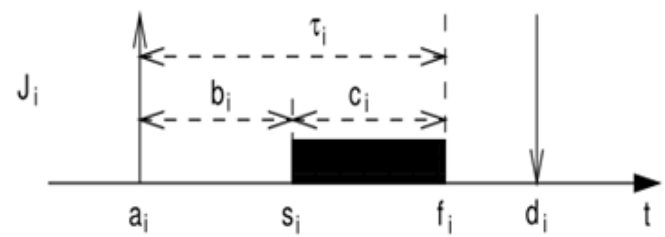

Figure 5: parameter of a real time network transmission task.

\begin{tabular}{|l|l|l|}
\hline & NETWORK SCHEDULING & CPU SCHEDULING \\
\hline TASK & Packet transmission & Task execution \\
\hline$h_{i}$ & Transmission period & Task period \\
\hline$c_{i}$ & Transmission time & Computation time \\
\hline$f_{i}$ & Finishing time & Finishing time \\
\hline$d_{i}$ & Transmission deadline & Task deadline \\
\hline$U$ & Network utilization & CPU Utilization \\
\hline Preemption & Non preemptive & Preemptive \\
\hline
\end{tabular}


We define the network utilization factor $\mathrm{U}$ of a set of NCS plants which require periodic transmission of their sensors message as

$U=\sum_{i=1}^{N} \frac{c_{i}}{h_{i}}$

\section{Rate Monotonic Scheduling For Nes}

Normally we propose the RATE MONOTONIC scheduling algorithm for prioritized, preemptive scheduling for a set of independent, periodic real time tasks. RM is a fixed priority assignment. It has been shown by Lou and Layland showed that RM is optimal among all fixed priority assignments in the sense that no other fixed priority algorithm can schedule a task set that cannot be scheduled by RM.

But for non preemptive, periodic task, the result is summarized in theorem

Theorem2: A set of $\mathrm{N}$ independent, non preemptive, periodic task are schedulable if all $\mathrm{i}=1, \ldots \mathrm{N}$

$\frac{c_{1}}{h_{1}}+\frac{c_{2}}{h_{2}}+\ldots \ldots+\frac{c_{i}}{h_{i}}+\frac{\bar{b}_{l, i}}{h_{i}} \leq i\left(2^{1 / i}-1\right)$

Where ${ }^{b_{l, i}}$ is task I's worst case blocking time by the lower priority tasks.

When a set of NCS plants are connected to the network and arbitrate for network bandwidth, based on the RM scheduling algorithm, a faster plant with higher transmission rate should be given priority over a slower plant.

\section{Problem Formulation In Scheduling Optimization:}

In the analysis of scheduling of NCSs using the RM scheduling algorithm, problem is how we can select an optimal feasible schedule which can minimize some performance measure? Assume each NCS is associated with a performance measure function, ${ }_{i}\left(h_{i}\right)$. Which gives the control cost as a function of transmission period $h_{i}$. Therefore, we can formulate the following optimimization problem:

minimize (maximize) $J_{i}\left(h_{i}\right)=\sum_{i=1}^{N} J_{i}\left(h_{i}\right)$

Subject to

RM schedulability constraints:

$h_{i} \leq \ldots \ldots \leq h_{N}$

$\frac{c_{1}}{h_{1}}+\ldots \ldots .+\frac{c_{i}}{h_{i}}+\frac{\bar{b}_{l, i}}{h_{i}} \leq i\left(2^{1 / i}-1\right), i=1, \ldots, N$;

NCS stability constraints:

$$
h_{i} \leq h_{\text {suff }, i}-\bar{b}_{i}, i=1, \ldots ., N
$$

The selection of the performance measure function $I_{i}\left(h_{i}\right)$ is crucial in the optimization problem. It directly relatesthe control cost to the NCS transmission period $h_{i}$.

Example: Performance Of Ncss- Non Ideal Transmission

Let the continuous open loop plant be

$\left[\begin{array}{l}\dot{x}_{1} \\ \bar{x}_{2}\end{array}\right]=\left[\begin{array}{cc}0 & 10 \\ 0 & 0\end{array}\right]\left[\begin{array}{l}x_{1} \\ x_{2}\end{array}\right]+\left[\begin{array}{ll}0 \\ 1\end{array}\right] u$

A continuous state feedback controller is $\mathrm{u}=-\mathrm{kx}$, where $\mathrm{K}=[500,100]$. Assume that the full state information is transmitted across a network. Now consider two version of the plant with different constant transmission periods: $h_{1}=0.008 s$ and $h_{2}=0.016 s$. Assume two system are periodic.

We assume the constant transmission time $c_{1}=c_{2}=0.003 s$. Now, consider the RM scheduling of the two NCSs on a network. By the RM algorithm, plant 1 gets higher priority since it has a higher transmission rate. Assume the two NCSs are in phase. Then NCS 1's data is delayed by its own transmission time $\tau=0.003 \mathrm{~s}$, while that of NCS 2 is delayed by its own transmission time plus pre-emption by the higher priority NCS 1 , $\tau=0.006 \mathrm{~s}$ , are shown in figure 6. 


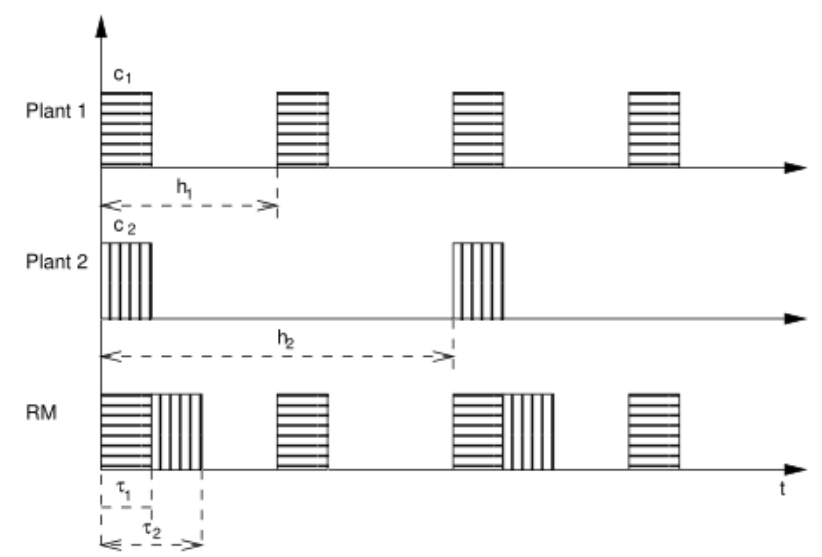

Figure 6: RM scheduling result

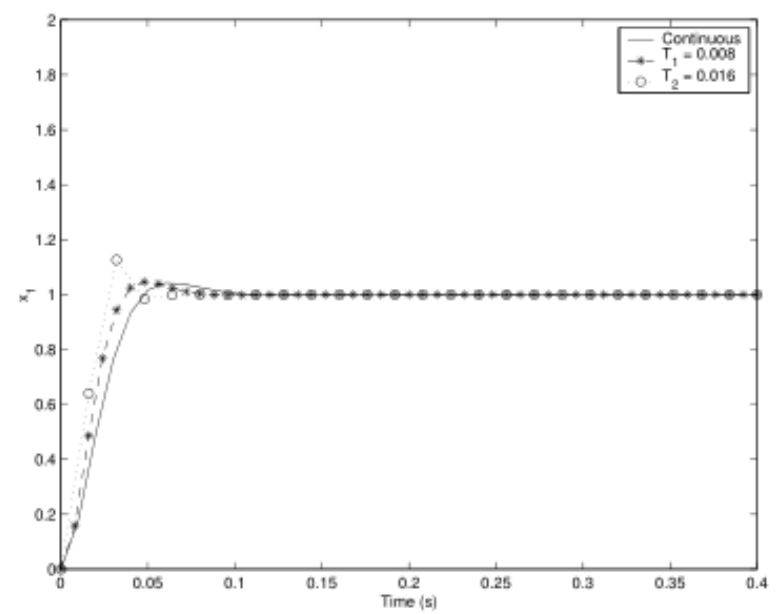

Figure 7: scaled step response of original control system

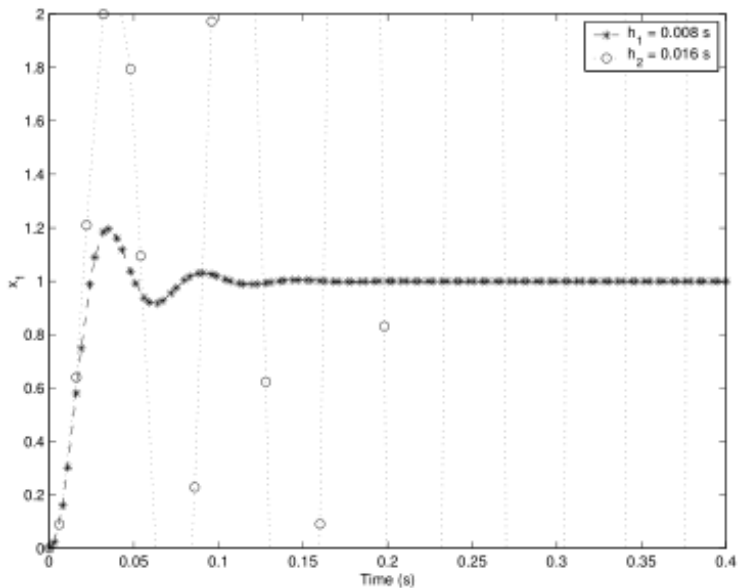

Figure 8: scaled step response of RM scheduling

The figure 7 shown the scaled step response of the original control system. The figure 8 shows the depicts the scaled step response when RM scheduling is employed and delays are taken in to account. With NCS 1 's response similar to the original one, NCS 2's response is unstable, caused by the excessive delay during the transmission.

The above example has demonstrated that scheduling and transmission delays degrade system performance and may cause system in stability. 


\section{Conclusion}

Scheduling analysis of RM scheduling has been carried out. We have formulated the optimal scheduling problem with the RM schedulability constraints and NCS stability constraints. The computation for simulating the plant and controller is carried out using MATLAB. We illustrate the result.

\section{References}

[1] K. Albers and F. Slomka, “An Event Stream Driven Approximation for the Analysis of Real-Time Systems,” Proc. 16th Euromicro Conf. Real-Time Systems, pp. 187-195, 2004.

[2] N.C. Audsley, A. Burns, M. Richardson, K.W. Tindell, and A.J. Wellings, "Applying New Scheduling Theory to Static Priority PreEmptive Scheduling," Software Eng. J., vol. 8, no. 5, pp. 284-292, 1993

[3] E. Bini and G.C. Buttazzo, "Measuring the Performance of Schedulability Tests," J. Real-Time Systems, vol. 30, nos. 1/2, pp. 129$154,2005$.

[4] G.C. Buttazzo, "Real-Time Scheduling and Resource Management," Handbook of Real-Time and Embedded Systems. Chapman \& Hall/CRC, 2008

[5] S. Chakraborty, S. Kunzli, and L. Thiele, “Approximate Schedulability Analysis,” Proc. IEEE Real-Time Systems Symp., pp. 159168

[6] S.K. Baruah, A.K. Mok, and L.E. Rosier, "Preemptively Scheduling Hard-Real-Time Sporadic Tasks on One Processor," Proc. 11thIEEE Real-Time System Symp., pp. 182-190, 1990.

[7] S.K. Baruah, L.E. Rosier, and R.R. Howell, "Algorithms and Complexity Concerning the Preemptive Scheduling of Periodic RealTime Tasks on One Processor,” J. Real-Time Systems, vol. 4, no. 2, pp. 301-324, 1990.

[8] S.K. Baruah, R.R. Howell, and L.E. Rosier, "Feasibility Problems for Recurring Tasks on One Processor," Theoretical Computer Science, vol. 118, pp. 3-20, 1993M Ozaki, Y. Adachi, Y. Iwahori, and N. Ishii, Application of fuzzy theory to writer recognition of Chinese characters, International Journal of Modelling and Simulation, 18(2), 1998, 112-116. (8) 